\title{
RADIATION THERAPY IS ASSOCIATED WITH AN INCREASED INCIDENCE OF CARDIAC EVENTS IN SMALL-CELL LUNG CANCER PATIENTS
}

\author{
Matthew J. Ferris, MD ${ }^{1,2}$, Renjian Jiang, MD, MPH $^{2,3}$, Madhusmita Behera, $\mathrm{PhD}^{2,4}$, Suresh \\ S. Ramalingam, $\mathbf{M D}^{2,4}$, Walter J. Curran, $\mathbf{M D}^{1,2}$, and Kristin A. Higgins, $\mathbf{M D}^{1,2}$ \\ ${ }^{1}$ Department of Radiation Oncology, Emory University Atlanta, Georgia \\ ${ }^{2}$ Winship Cancer Institute at Emory University, Atlanta, Georgia; \\ ${ }^{3}$ Department of Epidemiology, Rollins School of Public Health, Emory University, Atlanta, Georgia; \\ ${ }^{4}$ Department of Hematology and Medical Oncology, Emory University, Atlanta, Georgia
}

\section{Abstract}

Purpose: Cardiac radiation dose was a predictor of inferior overall survival (OS) in the nonsmall-cell lung cancer trial RTOG 0617. We examined the association between radiation therapy (RT) and cardiac events (CE) for small cell lung cancer (SCLC) patients.

\begin{abstract}
Methods and Materials: The US population-based Surveillance, Epidemiology, and End Results (SEER) Program and Medicare claims databases were queried for rates of CE among SCLC patients treated with chemotherapy (CTX) +/- RT. Propensity score matching (PSM) and multivariate analysis (MVA) were conducted. Patients were matched for actual/theoretical RT start date (to prevent immortal time bias) and then full PSM balanced clinical characteristics. Cumulative incidence function curves were generated.
\end{abstract}

Results: From 2000 - 2011, 7,060 patients were included: 2,892 limited-stage (LS-SCLC) and 4,168 extensive-stage (ES-SCLC). Grouping LS-SCLC and ES-SCLC together, the incidence of $\mathrm{CE}$ for the CTX + RT and CTX-only groups was 44.1 vs. $39.0 \%$ at 60 months $(P=0.008)$. After PSM (5,286 patients), the incidence of CE for the CTX + RT and CTX-only groups was 43.0 vs. $38.6 \%$ at 60 months $(P=0.033)$. Analysis of only LS-SCLC (2,016 patients) demonstrated the incidence of CE for CTX + RT vs. CTX-only groups was 50.3 vs. $42.0 \%$ at 60 months ( $P=$ 0.0231). MVA again demonstrated association between CE and RT (HR 1.20; 95\% CI $1.06-1.37$; $P=0.005)$. After PSM (1,614 patients), the incidence of CE for CTX + RT vs. CTX-only groups was 51.7 vs. $41.6 \%$ at 60 months $(P=0.0042)$.

Corresponding author: Matthew J. Ferris, MD, The Emory Clinic, 1365 Clifton Road NE, Atlanta, Georgia 30322, Tel 410-241-4504, Fax 404-778-3670, mjferri@emory.edu.

Publisher's Disclaimer: This is a PDF file of an unedited manuscript that has been accepted for publication. As a service to our customers we are providing this early version of the manuscript. The manuscript will undergo copyediting, typesetting, and review of the resulting proof before it is published in its final citable form. Please note that during the production process errors may be discovered which could affect the content, and all legal disclaimers that apply to the journal pertain.

Conflicts of Interest:

No conflicts to declare. 
Conclusions: SCLC patients are at significant risk of developing CE post-treatment; RT is associated with an absolute increase in the rate of CE at 5-years of approximately $5 \%$ for all SCLC patients, and up to $10 \%$ for LS-SCLC patients. Cardiac risk management and cardiac-sparing RT techniques should be further evaluated for SCLC patients.

\section{SUMMARY}

Among small-cell lung cancer patients, we found baseline rates of cardiac events are about $40 \%$ at 5 -years in the US population. Treatment with radiation therapy contributes to an absolute increase in this rate of cardiac events at 5-years of approximately $5 \%$ for all patients (limited-stage and extensive-stage both), and up to $10 \%$ for the limited-stage population.

\section{Keywords}

small-cell lung cancer; cardiac/heart dose; radiation therapy/effects; cardiac events/toxicity

\section{INTRODUCTION}

Cardiac morbidity has long been posited as a consequence of radiation therapy (RT) to thoracic targets. Initially the bulk of these reports originated in studies of long-term survivors of mediastinal RT for Hodgkin lymphoma, with differing estimations as to the extent of clinical significance-particularly as modern radiation techniques and doses emerged. ${ }^{1-5}$ Given the more favorable prognosis of Hodgkin lymphoma compared to lung cancer, longer-term data exists, and a recent report identifies a more than doubling in the risk of heart failure at 25 years for patients receiving radiation doses $26-30 \mathrm{~Gy}$, and a more than tripling for radiation doses $\geq 31 \mathrm{~Gy}$, compared to controls. ${ }^{6}$ With the more unfavorable survival seen in lung cancers, the extent to which cardiac morbidities would have an effect on clinical outcomes has historically been less appreciated. Correlative breast cancer studies, though, have suggested that coronary events likely do begin in a time frame relevant to advanced lung cancer patients. For example, Marks et al describe volume-dependent perfusion defects present in $40 \%$ of left-sided breast cancer patients within 2 years of RT. ${ }^{7}$ Darby et al demonstrate in a population of breast cancer patients a linear increase in major coronary events by 7.4\% per Gy of mean heart dose, with events beginning within the first 5 years of exposure. ${ }^{8}$ Additionally, Gomez et al found in a prospectively monitored group of patients who were estimated to receive $\geq 20 \mathrm{~Gy}$ to the heart during thoracic RT that changes in BNP and ECGs were notable actually during the RT course. ${ }^{9}$

Increasingly, radiation dose to the heart is being verified as a contributor to outcomes in patients who receive RT for non-small-cell lung cancer (NSCLC). ${ }^{10-17}$ Little data, however, quantifies cardiac morbidity of RT for small-cell lung cancer (SCLC) patients. Thoracic RT delivered concurrent with the first or second cycle of cisplatin and etoposide chemotherapy (CTX) is considered standard of care for limited-stage (LS) SCLC. ${ }^{18-20}$ Traditionally, per the seminal results from Turrisi et al, ${ }^{18}$ anticipated 5-year overall survival (OS) has been expected to be approximately $23 \%$, though this figure has been improved upon over time as demonstrated by the CONVERT trial, ${ }^{20}$ which describes 5 -year OS as $34 \%$ in the twicedaily radiation group versus $31 \%$ for the once-daily group. For extensive-stage (ES) SCLC, thoracic RT should be considered in patients who respond to chemotherapy, and Slotman et 
al report an improvement in 2-year OS to $13 \%$ from $3 \%$ with the addition of thoracic RT. 21,22

In the NSCLC trial Radiation Therapy Oncology Group (RTOG) 0617, heart $\mathrm{V}_{5}$ (volume of heart receiving $5 \mathrm{~Gy}$ ) and heart $\mathrm{V}_{30}$ were associated with increased risk of death, which persisted on multivariable analysis (MVA). ${ }^{16}$ Reporting on the long-term incidence of $\geq$ grade 3 cardiac events (CE) among a cohort of 125 patients enrolled on prospective RT trials for locally advanced NSCLC, Dess et al describe an incidence exceeding $10 \% .{ }^{12}$ Wang et al found heart dose to be independently associated with CE from a cohort of patients enrolled at their institution on prospective locally advanced NSCLC trials. ${ }^{15}$ Given the parallels in NSCLC, and especially with contemporary SCLC survival figures, normal tissue effects of RT such as cardiac toxicity gain importance in SCLC. With this study, we sought to examine the association between RT and CE for a large number of SCLC patients by utilizing the Surveillance, Epidemiology, and End Results (SEER) Program database and Medicare claims data.

\section{METHODS}

The SEER Program database and Medicare claims data were queried to establish rates of CE among SCLC patients treated with CTX $+/-$ RT. The SEER Program, funded by the National Cancer Institute, collects data from approximately $28 \%$ of the population of the United States, and the demographics are representative of the US population as a whole. ${ }^{23}$ Medicare currently serves approximately 55 million beneficiaries, the majority being age 65 or older, and is accepted by $91 \%$ of US physicians. ${ }^{24}$ While the SEER database provides useful information regarding oncologic diagnoses, staging, and survival, Medicare claims (billing) data—cross-linked to the SEER data by unique patient identifiers—provides an ability to assess incidence of CE, details of CTX and RT courses, and certain tumor characteristics. Approval for this study was obtained from both SEER and the Institutional Review Board of XXXX.

After including patients in the SEER database with a diagnosis of lung cancer, all patients less than 65 years of age or diagnosed before the year 2000 were excluded due to the inherent composition of the Medicare claims data and the need to cross-link the two databases. Additional exclusion criteria consisted of: histology other than SCLC; unclear staging information or month of diagnosis; did not receive CTX within the first five months following diagnosis; unclear RT record in SEER or lack of corresponding Medicare claims data; received RT but not within the first 8 months following diagnosis (suggests palliative RT); and lack of continuous enrollment in Medicare 12 months prior to or after diagnosis.

CE were defined as any cardiac diagnosis assigned to a patient with a pathophysiology potentially influenced by RT, including: acute myocardial infarction or any acute heart disease, cardiomyopathy, dysrhythmia, heart failure, and pericarditis. Valvular heart disease and chronic/pre-existing diagnoses including old myocardial infarction, angina pectoris, chronic ischemic heart disease, and chronic pulmonary heart disease were not counted as events. CE data points beyond 5 years were sparse, and so a 5-year upper time limit was set for survival assessments during analysis. 
Initially, an attempt was made to determine the influence of RT fractionation on CE (oncedaily versus twice-daily), however, fractionation could not be reliably discerned from Medicare encounter billing codes, and so this attempt was abandoned.

\section{Statistical Analysis}

Statistical analysis was conducted using SAS Version 9.4. The significance level was set at 0.05. Descriptive statistics were generated to summarize patient, disease, and treatment characteristics. To prevent immortal time bias, CTX-only patients were matched 1:1 to CTX + RT patients to account for start date of RT, with the first actual or theoretical RT start date taken as the beginning of follow-up. We randomly sampled CTX-only patients who had survival time (from diagnosis date) at least as long as the survival time from diagnosis date to RT start date of a corresponding CTX + RT patient to match the CTX + RT patient without replacement, and assign the CTX + RT patient's RT start date as the theoretical start date of the CTX-only patient in the matched pair. A second phase of propensity score matching (PSM) balanced demographic, tumor, and clinical differences to reduce treatment selection bias-SCLC patients receiving RT were inherently a healthier group with less advanced disease. It should be noted that a consequence of this second phase of PSM is that a disproportionate amount of the unhealthier CTX-only patients and the healthier RT patients are then excluded, which is necessary in order to create equally matched groups.

We repeated this matching process over two iterations. The first iteration incorporated all SCLC patients regardless of stage and was performed to preserve sample size between groups and to generalize the results to a larger population of patients. The second iteration analyzed only LS-SCLC patients and was performed to see if effects of RT are magnified among LS-SCLC patients given they more routinely receive RT and at higher doses. Corresponding American Joint Committee on Cancer (AJCC) TNM staging was utilized to convert staging to LS-SCLC versus ES-SCLC when necessary. Patients were matched for age at diagnosis, gender, race, year of diagnosis, Charlson/Deyo score, primary tumor laterality, primary tumor lobe location, and disease stage. Notably, Charlson/Deyo score incorporates cardiac comorbidities such as congestive heart failure and myocardial infarct thus it serves as a reasonable approximation of baseline cardiac risk. Covariates incorporated into the propensity scores were identical for the first iteration of PSM and second iteration of PSM except that the first iteration included overall stage (LS-SCLC vs. ES-SCLC) as a covariate while the second iteration (all patients LS-SCLC) instead incorporated AJCC TStage and AJCC N-Stage as the means to match for disease burden. The greedy matching algorithm was utilized for PSM.

The univariate association (UVA) between each covariate and study cohort was assessed using the Chi-square test for categorical covariates and ANOVA for numerical covariates. Covariates included receipt of RT, age, gender, race, year of diagnosis, Charlson/Deyo score, primary tumor location, primary tumor laterality, AJCC T-Stage, and AJCC N-Stage. Cox proportional hazards models and Fine-Gray subdistribution hazard models were utilized in MVA to determine the effect of tumor and RT covariates on OS and CE. Backward selection with an alpha level of removal of 0.2 was used. Kaplan-Meier curves were generated to compare survival between CTX-only and CTX + RT groups. Log-rank tests were performed 
to compare survival difference of the Kaplan-Meier curves. We also generated Kaplan-Meier curves to compare OS as stratified by tumor laterality. Cumulative incidence function curves were generated to compare $\mathrm{CE}$ between groups with OS as serving as a competing event. We also analyzed incidence of $\mathrm{CE}$ in the year prior to onset of RT, as a means of assessing baseline cardiac risk between groups. Cumulative incidence function curves were also generated to compare CE and OS in the CTX + RT groups stratified by tumor laterality and primary tumor location in the upper versus lower lung.

\section{RESULTS}

From 2000 - 2011, 9,621 SCLC patients were available. For Consolidated Standards of Reporting Trials (CONSORT) diagram outlining change in patient numbers by exclusion criteria, see Figure 1. Please see eTable 1 for patient and disease characteristics prior to matching.

\section{First iteration: All SCLC patients included (LS-SCLC + ES-SCLC)}

After initial matching for RT start date, 7,060 patients were available (2,561 were excluded): 2,892 (41.0\%) with LS-SCLC and 4,168 (59.0\%) with ES-SCLC. RT was associated with CE $(P=0.008)$, with cumulative incidence as follows for the CTX + RT and CTX-only groups: $36.4 \%(34.8-37.9)$ vs. $35.4 \%$ (33.8 - 36.9) at 12 months, and $44.1 \%(42.5-45.7)$ vs. $39.0 \%$ (37.4 - 40.6) at 60 months, respectively. Table 1 demonstrates distribution of CE types (first CE since start of follow-up) experienced among all 7,060 patients. Figure 2 demonstrates distribution of CE (first CE since start of follow-up) by number of years from treatment. Notably, in the year prior to RT, rates of CE were significantly higher in the CTXonly group (37.45\%) compared to the CTX + RT group (30.54\%), suggesting that the CTXonly group had higher baseline cardiac risk $(P<0.0001)$. Within the CTX + RT group, patients with a $\mathrm{CE}$ in the year prior to RT had a significantly elevated incidence $(\mathrm{P}<0.0001)$ of CE following RT, compared to patients who did not experience a cardiac event in the year prior to RT: $55.4 \%(52.4-58.2)$ vs. $28.0 \%(26.3-29.8)$ at 12 months, and $62.3 \%$ (59.3 $65.0)$ vs. $36.2 \%$ (34.3 - 38.1), respectively. Within the CTX + RT group, tumor laterality was not significantly associated with $\mathrm{CE}(P=0.9776)$, with cumulative incidence as follows for left-sided versus right-sided primaries: $37.0 \%(34.6-39.6)$ vs. $36.0 \%(34-38.2)$ at 12 months, and $43.9 \%(41.3-46.5)$ vs. $44.6 \%(42.5-46.0)$ at 60 months. Also within the CTX + RT group, tumor location in the upper versus lower lobe was not significantly associated with CE $(P=0.5054)$, with cumulative incidence as follows for upper versus lower lobe primaries: $36.6 \%(34.3-38.8)$ vs. $35.7 \%(33.0-38.5)$ at 12 months, and $44.8 \%(42.5-$ $47.1)$ vs. $43.5 \%$ (40.6 - 46.3) at 60 months. UVA of association with OS demonstrated that lower Charlson/Deyo score $(P<0.001)$ was associated with better OS while absence of treatment with RT, extensive stage disease, and male gender were associated with worse OS (all $P<0.001$ ). MVA of association with OS demonstrated LS disease (HR 0.60; $0.57-$ $0.64 ; P<0.001)$, and lower Charlson/Deyo comorbidity score $(P<0.001)$ were associated with better OS. Absence of treatment with RT (HR 1.29; 1.23 - 1.36; $P<0.001$ ), male gender (HR 1.19; $1.13-1.24 ; P<0.001$ ), and earlier year of diagnosis (HR 1.05; 1.00 $1.10 ; P<0.044)$ were associated with worse OS. Kaplan-Meier curves demonstrated statistically significant superior OS for the CTX + RT vs. CTX-only group: 35.0\% (33.4- 
$36.5)$ vs. $21.4 \%(20.1-22.8)$ at 12 months, and $6.6 \%(5.7-7.5)$ vs. $2.3 \%(1.9$ vs. 2.9$)$ at 60 months, respectively. Kaplan-Meier curves stratified by tumor laterality demonstrated borderline statistical significance for left-sided primaries having worse OS compared to right-sided primaries $(P=0.059)$ with OS for left-sided primaries $27.1 \%$ (95\% CI 25.5 $28.7)$ at 12 months and $3.9 \%(3.2-4.8 \%)$ at 60 months versus OS for right-sided primaries $29.3 \%(27.9-30.8)$ at 12 months and $4.8 \%(4.1-5.5 \%)$ at 60 months. MVA of association with CE demonstrated higher hazard of CE for ES disease $(P<0.001)$, black race $(P<$ 0.001), and increasing Charlson/Deyo score from 0 (Charlson/Deyo score 1 HR 1.14; 1.04 1.24; $P=0.004$; and Charlson/Deyo score 2+ HR 1.77; $1.63-1.93 ; P<0.001)$. RT was not significantly associated with CE on MVA prior to PSM (HR 1.06; 95\% CI $0.98-1.14 ; P=$ $0.123)$.

After full PSM, 5,286 patients were included. Please see (eTable 2) for PSM balance check and distribution between groups; groups were well-matched. RT was significantly associated with CE $(P=0.033)$ after PSM, with cumulative incidence of CE for the CTX + RT and CTX-only groups: $36.3 \%$ (95\% CI $34.4-38.1)$ vs. $34.8 \%(33.0-35.5)$ at 12 months, and $43.0 \%(41.2-44.9)$ vs. $38.6 \%$ (36.6 - 40.3) at 60 months, respectively. Figure 3

demonstrates cumulative incidence function curves for $\mathrm{CE}$ and $\mathrm{OS}$ as stratified by treatment +/- RT, for prior to full PSM (Figure 3A, groups matched for RT start date only) and after full PSM (Figure 3B). As demonstrated in Figure 3, the cumulative incidence function difference for OS (between +/-RT groups) is reduced after PSM (Figure 3B) compared to before PSM (Figure 3A), indicating that the effect of potential OS advantage of RT on the incidence of CE is fully adjusted with PSM-which is reinforced by eTable 2 demonstrating no differences between matched groups. Additionally, the CE difference between CTX + RT and CTX-only groups remains stable before and after PSM, again indicating that the CE difference is not mainly due to the difference in patient baseline characteristics. Again, in the year prior to RT, rates of CE were significantly higher in the CTX-only group (34.54\%) compared to the CTX + RT group (30.72\%), suggesting that the CTX-only group had higher baseline cardiac risk $(P=0.0003)$.

\section{Second iteration: Only LS-SCLC patients included}

After initial matching for RT start date, 2,016 LS-SCLC patients were available. RT was again significantly associated with $\mathrm{CE}(P=0.0231)$, with cumulative incidence as follows for the CTX + RT and CTX-only groups: $39.9 \%(36.9-42.9)$ vs. $38.0 \%(35.0-40.9)$ at 12 months, and $50.3 \%(47.2-53.3)$ vs. $42.0 \%(39.0-44.7)$ at 60 months, respectively. In the year prior to RT, rates of CE were significantly higher in the CTX-only group (41.47\%) compared to the CTX + RT group (28.67\%), suggesting that the CTX-only group had higher baseline cardiac risk $(P<0.0001)$. Within the $\mathrm{CTX}+\mathrm{RT}$ group, patients with a $\mathrm{CE}$ in the year prior to RT had a significantly elevated incidence $(\mathrm{P}<0.0001)$ of CE following RT, compared to patients who did not experience a cardiac event in the year prior to RT: $60.9 \%$ (55.1 - 66.2) vs. $31.4 \%(28.1-34.8)$ at 12 months, and $69.1 \%(63.4-74.0)$ vs. $42.7 \%$ (39.1 - 46.4), respectively. Within the CTX + RT group, tumor laterality was not significantly associated with $\mathrm{CE}(P=0.6227)$, with cumulative incidence as follows for left-sided versus right-sided primaries: $40.4 \%(35.7-45.0)$ vs. $39.4 \%(35.4-43.3)$ at 12 months, and $51.03 \%(46.1-55.3)$ vs. $49.6 \%(45.4-53.5)$ at 60 months. Also within the CTX + RT 
group, tumor location in the upper versus lower lobe was not significantly associated with CE $(P=0.2006)$, with cumulative incidence as follows for upper versus lower lobe primaries: $40.8 \%(36.6$ - 44.9) vs. $36.8 \%(31.6-42.0)$ at 12 months, and 51.0\% (46.7 $55.2)$ vs. $47.0 \%(41.5-52.3)$ at 60 months. For UVA associations with OS, please see eTable 3. For MVA associations with OS, please see eTable 4. MVA of associations with CE demonstrated significant associations for treatment with RT (HR 1.20; 95\% CI $1.06-1.37$; $P=0.005$ ), gender (female gender HR 0.84; $0.74-0.95 ; P=0.005$ ), and Charlson/Deyo score of 2+ compared to 0 (Charlson/Deyo score 1 HR 1.13; 0.97 - 1.32; P 0.109; and Charlson/Deyo score 2+ HR 1.81; $1.56-2.11 ; \mathrm{P}<0.001)$.

After full PSM, 1,614 patients were included. Please see eTable 5 for PSM balance check and distribution between groups; groups were well-matched. RT was again significantly associated with CE $(P=0.0042)$, with cumulative incidence as follows for the CTX + RT and CTX-only groups: $40.8 \%(37.4-44.1)$ vs. $37.4 \%$ (34.1 - 40.7) at 12 months, and 51.7\% $(48.2-54.0)$ vs. $41.6 \%$ (38.3 - 44.9) at 60 months, respectively. Please see Figure 4 for cumulative incidence function curves for prior to full PSM (Figure 4A) and after full PSM (Figure 4B). Again, in the year prior to RT, rates of CE were significantly higher in the CTX-only group (39.54\%) compared to the CTX + RT group (30.36\%), suggesting that the CTX-only group had higher baseline cardiac risk $(P=0.0001)$.

In further analysis of all LS-SCLC patients who received RT, tumor laterality $(P=0.84)$ and RT modality $(P=0.62)$ were not associated with CE-though a low number of patients were treated with intensity-modulated (204) compared to 3D conformal $(2,960)$ RT $(1: 15$ ratio).

\section{DISCUSSION}

The impact of $\mathrm{CE}$ in lung cancer came to the forefront with the unexpected inferior survival of the dose-escalated arm of RTOG 0617. ${ }^{16}$ While other authors have been inconsistent in finding an association between heart dose and survival in other NSCLC patient populations, 25,26 a secondary dosimetric analysis of RTOG 0617 demonstrated that heart $\mathrm{V}_{40}$ was an independent predictor of inferior overall survival (OS). This analysis also demonstrated that IMRT was capable of producing lower heart doses, and concluded IMRT should be routinely considered to spare cardiac dose. ${ }^{11}$ Wang et al, in an analysis of 127 patients enrolled on prospective dose-escalation trials at their institution, also demonstrated that $\mathrm{CE}$ are common after high-dose thoracic RT and are associated with heart dose and baseline cardiac risk; they too conclude that CE in locally advanced NSCLC likely occur earlier than historical estimates. ${ }^{15}$ Spiers et al associated increasing heart volume and heart $\mathrm{V}_{50}$ with both cardiac toxicity and OS (that persisted with MVA) among 332 patients treated at a single institution for locally advanced NSCLC. ${ }^{14}$ Tucker et al describe in a large cohort— 468 patients from multiple institutions - an association between OS and mean heart dose and heart V5, though neither remained significant with MVA. ${ }^{25}$ Finally, a meta-analysis of 25 randomized doseescalation trials for NSCLC also demonstrated higher RT doses, when delivered concurrent with CTX, resulted in worse OS; increased toxicity being the implied culprit of this. ${ }^{10}$ Preexisting cardiac disease likely increases toxicity rates. ${ }^{12,15}$ 
Our study is novel in that it is the first to describe rates of cardiac toxicity associated with RT in SCLC. We found an approximate 5 percent absolute increase in the rate of CE at 5years experienced by all SCLC patients who receive RT compared to all SCLC patients who do not receive RT. On the first iteration of our analysis (both LS-SCLC and ES-SCLC patients included), treatment with RT did not persist as significant on MVA prior to full PSM. One possible explanation for this might be that since death is a competing risk with $\mathrm{CE}$, prior to full PSM there were relatively more patients who died in the CTX-only group (because of survival advantage of RT) compared to after PSM. Since death in the CTX-only group prevents $\mathrm{CE}$ from accruing (an individual is removed from the risk set when the individual experiences a competing event), the denominator in the CTX-only group is reduced and consequently the cumulative incidence of CE is increased-thus, the CE difference is less significant on the MVA prior to PSM. Survival advantage for RT was well controlled with PSM and the significant impact of RT on CE we believe should be considered genuine even with RT patients having better OS (and thus more time to accrue $\mathrm{CE})$. To that effect, our data is important in that it demonstrates this significant increase in the rate of CE in a group of patients-the ones receiving RT-that is inherently healthier at baseline. This is also reinforced by our analysis that demonstrated the CTX-only groups had higher rates of $\mathrm{CE}$ in the year prior to RT - thus were predisposed to higher cardiac risk. Furthermore, this 5\% at 5-years estimate is likely low, as it is not possible to discern RT target site using SEER or Medicare claims, and as such a small proportion of patients included in the RT group may have received RT not to the thorax at all but to a different site such as the brain (for prophylactic cranial radiation or treatment of brain metastases). For the second iteration of our analysis, we demonstrated an approximate 10 percent absolute increase in the rate of CE at 5-years experienced by LS-SCLC patients who are treated with RT compared to LS-SCLC patients who are not treated with RT. This magnification of the effect of RT is likely due to higher doses utilized during treatment. Again, the higher incidence of CE was demonstrated for the CTX + RT group, even despite the lower baseline cardiac risk that can be inferred by our analysis demonstrating a higher incidence of $\mathrm{CE}$ for the CTX-only group in the year prior to RT.

Beyond the added risk of CE contributed to by RT, this study also demonstrates the high prevalence of CE in the SCLC population as a whole. Even among patients not treated with RT, the rate of CE at 5-years appears to be approximately $40 \%$. This high rate of CE is likely a culmination of the patients' malignancies, age, socioeconomic circumstances and demographic characteristics including race, ${ }^{27,28}$ and-in many instances-years of smoking. The risk of CE is potentially further increased for SCLC compared to NSCLC due to the central location of many SCLCs and the frequently higher radiation doses utilized (66 to 70 Gy with once-daily fractionation, as opposed to $60 \mathrm{~Gy}$ for NSCLC). Routine involvement of a cardiologist in the post-treatment care of these patients may be a reasonable consideration, and statin or ACE inhibitor therapy ${ }^{29}$ should perhaps be further evaluated as a regular component of management. Our study also has now contributed to the literature that suggests that baseline cardiac risk increases the incidence of CE with RT, ${ }^{12,15}$ as we demonstrated that within the CTX + RT groups there were significantly higher incidences of $\mathrm{CE}$ in the patients that experienced a $\mathrm{CE}$ in the year prior to RT (a surrogate for patients with higher baseline risk) compared to patients that did not. 
This study is limited by the inherent weaknesses of retrospective data, of which the SEER database and Medicare claims data are composed. Additionally, analysis was limited to patients 65 years of age or older, as described above, and patients treated at a younger age may be at lower risk of CE. Given that two thirds of lung cancer diagnoses are in patients 65 or older, though, our study population is reasonably representative of the lung cancer population as a whole. ${ }^{30}$ Unfortunately, lack of consistent documentation in Medicare billing codes distinguishing once-daily versus twice-daily RT regimens prevented us from proceeding with analysis of the impact of fractionation on the development of $\mathrm{CE}$. Use of twice-daily fractionation in a portion of LS-SCLC patients is one factor that precludes a direct extrapolation of the CE rates suggested by NSCLC analyses to SCLC patients-it remains unclear how twice-daily fractionation might affect cardiac toxicity. The nature of the data inherent to this analysis precluded a precise evaluation of the effect of heart dose on the incidence of CE, as RT plans were not available for review. As a surrogate for heart dose, we attempted analyses of the incidence of CE within the CTX + RT groups as stratified by tumor laterality and upper vs. lower lobe tumors, and did not demonstrated an effect for either. Since no effect was demonstrated, it seems that laterality and lobe location as coded in these databases probably do not function as adequate surrogates of heart dose, as in reality heart dose is a culmination of numerous factors including primary tumor location, involved node location, disease size, target volume expansion, and treatment technique. While our data did not suggest an impact of RT modality (IMRT vs. 3DCRT) on the development of $\mathrm{CE}$, the available population was limited by low numbers of patients receiving IMRT compared to 3DCRT. We do believe that use of highly conformal modern RT techniques such as IMRT — which have the inherent ability to spare cardiac dose — are likely to contribute to clinically significant reductions in cardiac toxicities from RT. In summary, we believe cardiac risk factors should be optimized post-treatment, and means of minimizing the development of cardiac events should be further investigated, whether via pharmacotherapy or advanced radiation techniques.

\section{CONCLUSIONS}

In this large US population-based database study, we demonstrated that RT is associated with an absolute increase in the rate of CE at 5-years of approximately 5\% for all SCLC patients, and up to $10 \%$ for LS-SCLC patients. We believe our data demonstrates that RT contributes to a clinically significant increase in the incidence of cardiac events, and in a relatively early time frame that is relevant for many SCLC patients.

\section{Supplementary Material}

Refer to Web version on PubMed Central for supplementary material.

\section{ACKNOWLEDGMENTS}

This study used the linked SEER-Medicare database. The interpretation and reporting of these data are the sole responsibility of the authors. The authors acknowledge the efforts of: the Applied Research Program; the National Cancer Institute; the Office of Research, Development, and Information, CMS; Information Management Services, Inc.; and the SEER tumor registries in the creation of the SEER-Medicare database. 


\section{Abbreviation List:}

$\begin{array}{ll}\text { RT } & \text { Radiation therapy } \\ \text { NSCLC } & \text { Non-small-cell lung cancer } \\ \text { SCLC } & \text { Small-cell lung cancer } \\ \text { CTX } & \text { Chemotherapy } \\ \text { LS } & \text { Limited-stage } \\ \text { OS } & \text { Overall survival } \\ \text { ES } & \text { Extensive-stage } \\ \text { RTOG } & \text { Radiation Therapy Oncology Group } \\ \text { MVA } & \text { Multivariable analysis } \\ \text { CE } & \text { Cardiac events } \\ \text { SEER } & \text { Surveillance, Epidemiology, and End Results } \\ \text { UVA } & \text { Univariate association } \\ \text { PSM } & \text { Propensity score matching } \\ \text { AJCC } & \text { American Joint Committee on Cancer } \\ \text { CONSORT } & \text { Consolidated Standards of Reporting Trials } \\ \end{array}$

\section{REFERENCES}

1. King V, Constine LS, Clark D, et al. Symptomatic coronary artery disease after mantle irradiation for Hodgkin's disease. Int J Radiat Oncol Biol Phys 1996;36:881-9. [PubMed: 8960517]

2. Galper SL, Yu JB, Mauch PM, et al. Clinically significant cardiac disease in patients with Hodgkin lymphoma treated with mediastinal irradiation. Blood 2011;117:412-8. [PubMed: 20858859]

3. Savage DE, Constine LS, Schwartz RG, Rubin P. Radiation effects on left ventricular function and myocardial perfusion in long term survivors of Hodgkin's disease. Int J Radiat Oncol Biol Phys 1990;19:721-7. [PubMed: 2211221]

4. Glanzmann C, Kaufmann P, Jenni R, Hess OM, Huguenin P. Cardiac risk after mediastinal irradiation for Hodgkin's disease. Radiother Oncol 1998;46:51-62. [PubMed: 9488128]

5. Constine LS, Schwartz RG, Savage DE, King V, Muhs A. Cardiac function, perfusion, and morbidity in irradiated long-term survivors of Hodgkin's disease. Int J Radiat Oncol Biol Phys 1997;39:897-906. [PubMed: 9369139]

6. van Nimwegen FA, Ntentas G, Darby SC, et al. Risk of heart failure in survivors of Hodgkin lymphoma: effects of cardiac exposure to radiation and anthracyclines. Blood 2017;129:2257-65. [PubMed: 28143884]

7. Marks LB, Yu X, Prosnitz RG, et al. The incidence and functional consequences of RT-associated cardiac perfusion defects. Int J Radiat Oncol Biol Phys 2005;63:214-23. [PubMed: 16111592]

8. Darby SC, Ewertz M, McGale P, et al. Risk of ischemic heart disease in women after radiotherapy for breast cancer. N Engl J Med 2013;368:987-98. [PubMed: 23484825] 
9. Gomez DR, Yusuf SW, Munsell MF, et al. Prospective exploratory analysis of cardiac biomarkers and electrocardiogram abnormalities in patients receiving thoracic radiation therapy with high-dose heart exposure. J Thorac Oncol 2014;9:1554-60. [PubMed: 25521400]

10. Ramroth J, Cutter DJ, Darby SC, et al. Dose and Fractionation in Radiation Therapy of Curative Intent for Non-Small Cell Lung Cancer: Meta-Analysis of Randomized Trials. Int J Radiat Oncol Biol Phys 2016;96:736-47. [PubMed: 27639294]

11. Chun SG, Hu C, Choy H, et al. Impact of Intensity-Modulated Radiation Therapy Technique for Locally Advanced Non-Small-Cell Lung Cancer: A Secondary Analysis of the NRG Oncology RTOG 0617 Randomized Clinical Trial. J Clin Oncol 2017;35:56-62. [PubMed: 28034064]

12. Dess RT, Sun Y, Matuszak MM, et al. Cardiac Events After Radiation Therapy: Combined Analysis of Prospective Multicenter Trials for Locally Advanced Non-Small-Cell Lung Cancer. J Clin Oncol 2017;35:1395-402. [PubMed: 28301264]

13. Jan N, Guy C, Reshko LB, Hugo GD, Weiss E. Lung and Heart Dose Variability During Radiation Therapy of Non-Small Cell Lung Cancer. Int J Radiat Oncol Biol Phys 2017;98:683-90. [PubMed: 28581410]

14. Speirs CK, DeWees TA, Rehman S, et al. Heart Dose Is an Independent Dosimetric Predictor of Overall Survival in Locally Advanced Non-Small Cell Lung Cancer. J Thorac Oncol 2017;12:293301. [PubMed: 27743888]

15. Wang K, Eblan MJ, Deal AM, et al. Cardiac Toxicity After Radiotherapy for Stage III Non-SmallCell Lung Cancer: Pooled Analysis of Dose-Escalation Trials Delivering 70 to 90 Gy. J Clin Oncol 2017;35:1387-94. [PubMed: 28113017]

16. Bradley JD, Paulus R, Komaki R, et al. Standard-dose versus high-dose conformal radiotherapy with concurrent and consolidation carboplatin plus paclitaxel with or without cetuximab for patients with stage IIIA or IIIB non-small-cell lung cancer (RTOG 0617): a randomised, two-bytwo factorial phase 3 study. Lancet Oncol 2015;16:187-99. [PubMed: 25601342]

17. Stam B, van der Bijl E, van Diessen J, et al. Heart dose associated with overall survival in locally advanced NSCLC patients treated with hypofractionated chemoradiotherapy. Radiother Oncol 2017;125:62-5. [PubMed: 28939179]

18. Turrisi AT, 3rd, Kim K, Blum R, et al. Twice-daily compared with once-daily thoracic radiotherapy in limited small-cell lung cancer treated concurrently with cisplatin and etoposide. N Engl J Med 1999;340:265-71. [PubMed: 9920950]

19. Ettinger DS, Berkey BA, Abrams RA, et al. Study of paclitaxel, etoposide, and cisplatin chemotherapy combined with twice-daily thoracic radiotherapy for patients with limited-stage small-cell lung cancer: a Radiation Therapy Oncology Group 9609 phase II study. J Clin Oncol 2005;23:4991-8. [PubMed: 15939930]

20. Faivre-Finn C, Snee M, Ashcroft L, et al. Concurrent once-daily versus twice-daily chemoradiotherapy in patients with limited-stage small-cell lung cancer (CONVERT): an openlabel, phase 3, randomised, superiority trial. Lancet Oncol 2017.

21. Slotman BJ, van Tinteren H, Praag JO, et al. Use of thoracic radiotherapy for extensive stage smallcell lung cancer: a phase 3 randomised controlled trial. Lancet 2015;385:36-42. [PubMed: 25230595]

22. Jeremic B, Shibamoto Y, Nikolic N, et al. Role of radiation therapy in the combined-modality treatment of patients with extensive disease small-cell lung cancer: A randomized study. J Clin Oncol 1999;17:2092-9. [PubMed: 10561263]

23. National Cancer Institute (U.S.): Surveillance E, and End Results Program. Overview of the SEER Program. Accessed 11 Jul 2017: https://seer.cancer.gov.

24. Altman D, Frist WH. Medicare and Medicaid at 50 Years: Perspectives of Beneficiaries, Health Care Professionals and Institutions, and Policy Makers. JAMA 2015;314:384-95. [PubMed: 26219056]

25. Tucker SL, Liu A, Gomez D, et al. Impact of heart and lung dose on early survival in patients with non-small cell lung cancer treated with chemoradiation. Radiother Oncol 2016;119:495-500. [PubMed: 27189523] 
26. Guberina M, Eberhardt W, Stuschke M, et al. Heart dose exposure as prognostic marker after radiotherapy for resectable stage IIIA/B non-small-cell lung cancer: secondary analysis of a randomized trial. Ann Oncol 2017;28:1084-9. [PubMed: 28453703]

27. Nadruz W, Jr., Claggett B, Henglin M, et al. Widening Racial Differences in Risks for Coronary Heart Disease. Circulation 2018;137:1195-7. [PubMed: 29530895]

28. Van Dyke M, Greer S, Odom E, et al. Heart Disease Death Rates Among Blacks and Whites Aged >/=35 Years - United States, 1968-2015. MMWR Surveill Summ 2018;67:1-11.

29. van der Veen SJ, Ghobadi G, de Boer RA, et al. ACE inhibition attenuates radiation-induced cardiopulmonary damage. Radiother Oncol 2015;114:96-103. [PubMed: 25465731]

30. American Cancer Society. Small Cell Lung Cancer. Accessed 11 Jul 2017 at: https://cancer.org/ cancer/small-cell-lung-cancer/about/key-statistics.html. 
459,787 SEER lung cancer cases

450,166 Patients excluded

102,854 Patients with a previous or concurrent malignancy

74,190 Patients $<65$ years of age (no Medicare claims data available)

856 Patients diagnosed prior to year 2000 (no Medicare claims data available)

8,557 Patients diagnosed only at time of death certificate/autopsy

242,069 Patients with lung cancer histology other than small-cell

4,386 Patients with unclear staging information (presence/absence of metastases)

16 Patients with unknown month of diagnosis

15,037 Patients who did not receive chemotherapy

588 Patients who did not receive chemotherapy within first 5 months after diagnosis

566 Patients with unclear radiation record in SEER or in corresponding Medicare data

149 Patients who received radiation but not within first 0-8 months (suggests palliation)

898 Patients not continuously enrolled in Medicare during follow-up period

9,621 Cases included prior to matching

Figure 1.

Consolidated Standards of Reporting Trials (CONSORT) diagram.

Abbreviations: SEER $=$ Surveillance, Epidemiology, and End Results database 


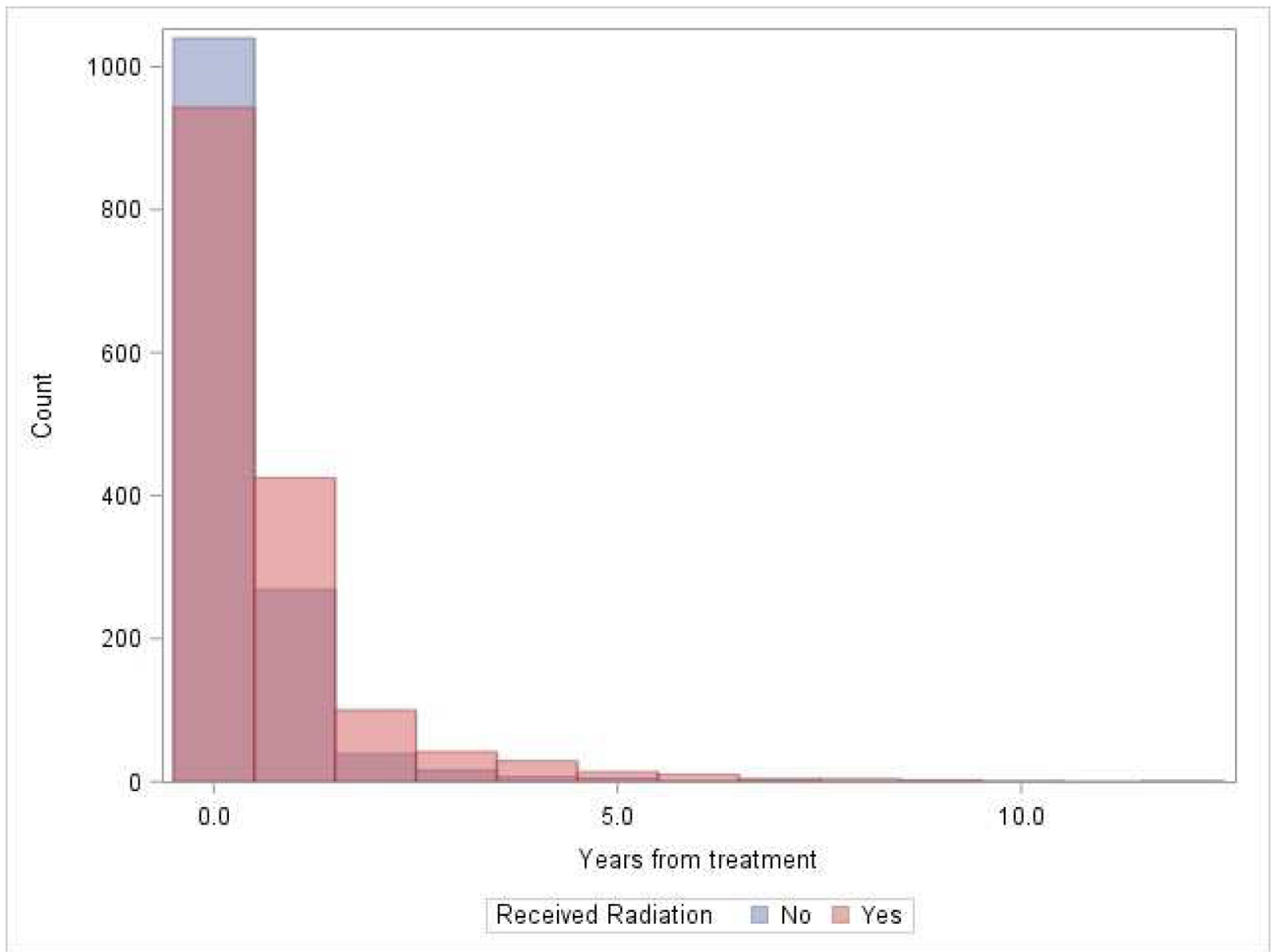

Figure 2.

Distribution of cardiac events by number of years from treatment 

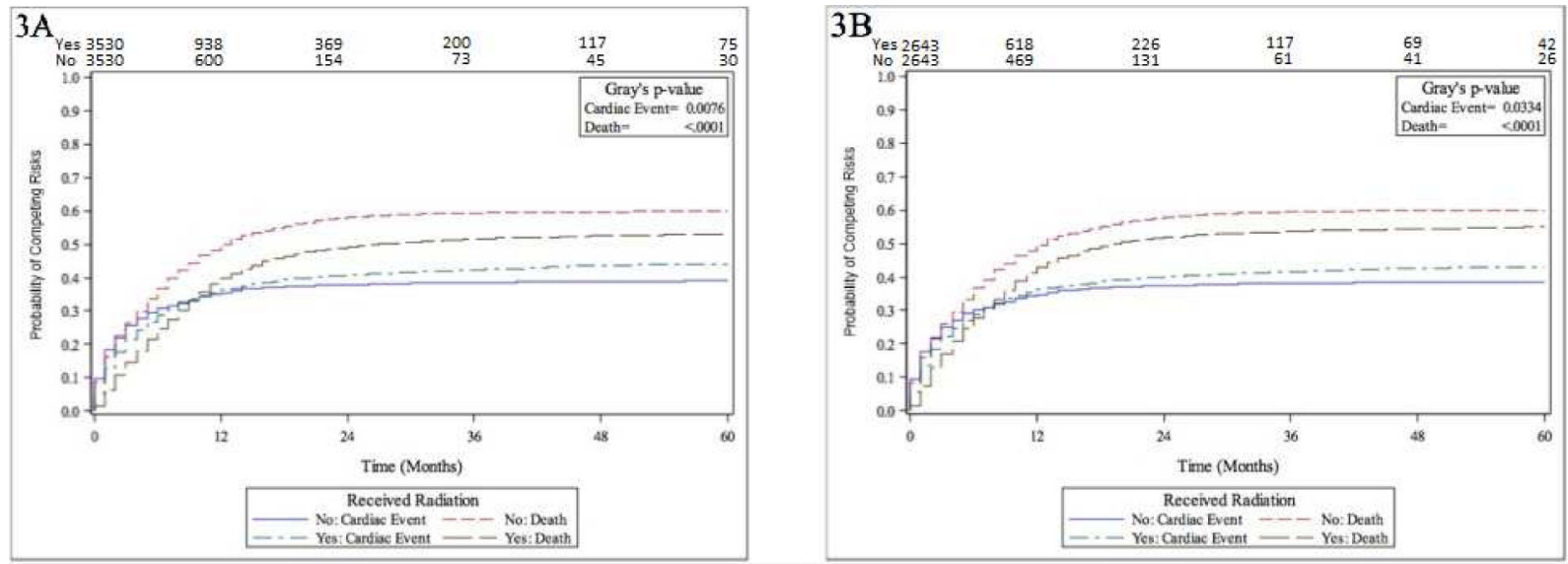

Figure 3.

Numbers-at-risk for cardiac events shown on x-axis. Figure 3A. Cumulative incidence function curves for death and cardiac events stratified by $+/$-radiation therapy (combined limited-stage and extensive-stage analysis, prior to propensity score matching) Figure 3B. Cumulative incidence function curves for death and cardiac events stratified by $+/$-radiation therapy (combined limited-stage and extensive-stage analysis, after propensity score matching) 

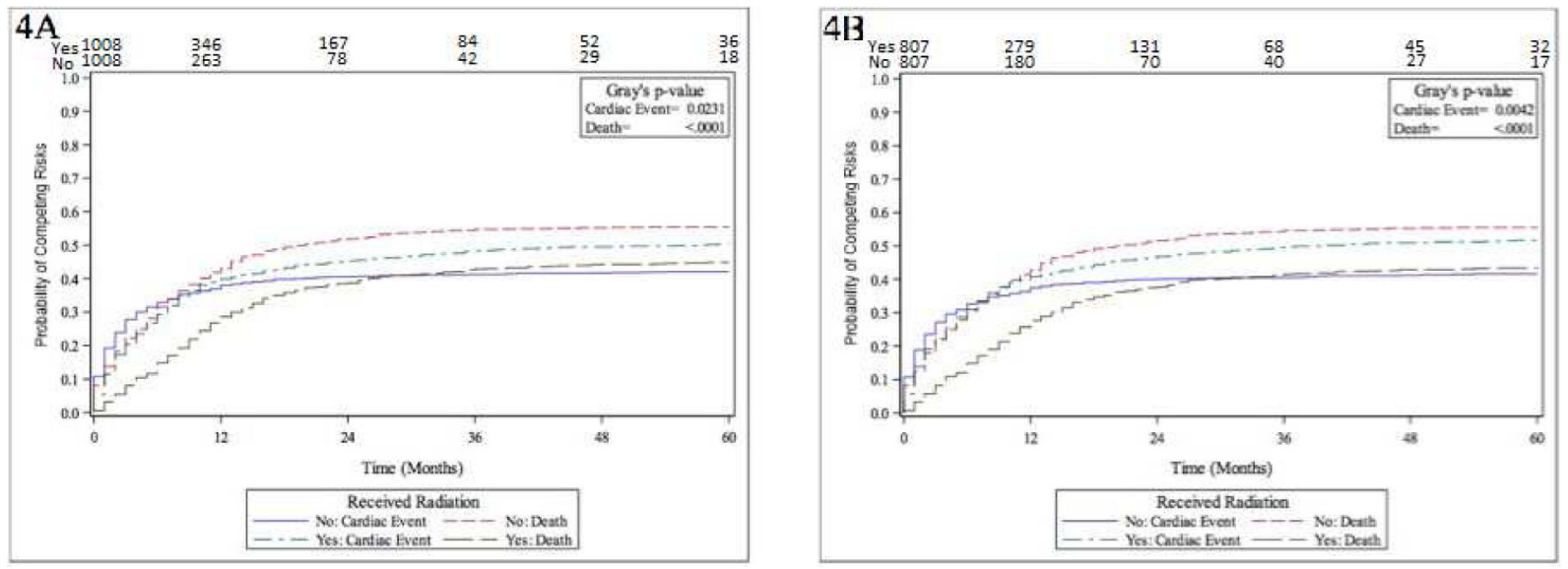

Figure 4.

Numbers-at-risk for cardiac events shown on x-axis. Figure 4A. Cumulative incidence function curves for death and cardiac events stratified by $+/$-radiation therapy (limited-stage analysis, prior to propensity score matching) Figure 4B. Cumulative incidence function curves for death and cardiac events stratified by +/-radiation therapy (limited-stage analysis, after propensity score matching) 
Table 1.

Distribution of cardiac event types $(n=7,060)$

\begin{tabular}{lcc}
\hline \multicolumn{1}{c}{ Event type } & Frequency & Percent \\
\hline No cardiac event experienced & 4,105 & 58.14 \\
Acute heart disease & 545 & 7.72 \\
Acute myocardial infarction & 258 & 3.65 \\
Cardiomyopathy & 215 & 3.05 \\
Dysrhythmia & 156 & 2.21 \\
Heart failure & 1,459 & 20.67 \\
Pericarditis & 322 & 4.56 \\
\hline
\end{tabular}

